\title{
Simulations of Halo Supernova Remnants
}

\author{
R.L. Shelton \\ Laboratory for High Energy Astrophysics, NASA/Goddard Space Flight Center, \\ Greenbelt, MD 20706, USA.
}

\begin{abstract}
High latitude observations find $\mathrm{C}^{3+}, \mathrm{N}^{4+}$, and $\mathrm{O}^{5+}$, while shadowing by intervening hydrogen clouds indicates the presence of soft $\mathrm{X}$-ray emitting gas in the halo of our galaxy. This paper reports on detailed hydrocode simulations of one of the possible source types: isolated supernova remnants evolving in the tenuous ambient medium of the halo. Their $\mathrm{O}^{5+}$ column densities are similar to those observed from the halo. The $\mathrm{N}^{4+}, \mathrm{C}^{3+}$, and X-ray properties are also simulated and discussed. The calculations may also be of interest for the Local Bubble, external galaxies, and non-thermal pressure estimations.
\end{abstract}

\section{Introduction}

The high latitude observations of $\mathrm{C}^{3+}, \mathrm{N}^{4+}, \mathrm{O}^{5+}$, and soft $\mathrm{X}$-rays are not completely explained, though several scenarios have been proposed and modeled. Multiple mechanisms may be at play and all should be considered. One of the possible contributors is the collection of isolated supernova remnants (SNRs) evolving in the halo. The simulations presented in this paper show that they harbor hot gas and $\mathrm{UV}$ ions $\left(\mathrm{C}^{3+}, \mathrm{N}^{4+}\right.$, and $\left.\mathrm{O}^{5+}\right)$ for nearly $2 \times 10^{7}$ $\mathrm{yrs}^{1}$. The supernova rates from Ferrière (1995) give about one supernova per 600,000 years per $\mathrm{kpc}^{2}$ above $z \geq 300$ pc at our galactocentric ${ }^{2}$ radius. Combining the rate with the lifetime yields about 30 halo SNRs per $\mathrm{kpc}^{2}$, a number which is high enough to make them relevant to our understanding of the halo. Previously, Ferrière (1995) and Cioffi (1991) modeled halo SNRs. The simulations presented in this paper differ from those in method and breadth of predictions.

This paper is focused on characterizing the $\mathrm{O}^{5+}, \mathrm{N}^{4+}$, and $\mathrm{C}^{3+}$ and $\mathrm{X}$-ray properties of isolated SNRs evolving in the halo. The main results are that the halo supernova remnants harbor much $\mathrm{O}^{5+}$, and may explain roughly $3 / 4$ ths of that expected to reside in the halo. The average $\mathrm{N}^{4+}$ and $\mathrm{C}^{3+}$ column densities are lower. The young SNRs are X-ray bright, but the fluxes averaged over their entire lifetimes are much smaller. Isolated SNRs fill a very small fraction of the halo.

${ }^{1}$ Merging with the ambient gas does not shorten their lifetimes.

${ }^{2}$ A cone having a $50^{\circ}$ angle from the zenith and truncated below $z=300 \mathrm{pc}$ also has about one supernova every 600,000 years. 


\section{Modeling}

Halo SNRs were simulated with a detailed, one-dimensional hydrodynamic computer code (Shelton, 1996) employing non-equilibrium ionization and recombination rates, thermal conduction, and magnetic pressure. The (unmodeled) density gradient should have minimal effect because the hot bubble diameters are $\sim 1 / 3$ of the density scale height and Maciejewski et al. (1996) found that SNRs evolving in moderate density gradients are nearly spherical.

The primary computer run used an explosion energy of $5 \times 10^{50} \mathrm{ergs}$, ambient density $(n)$ of 0.01 atoms $\mathrm{cm}^{-3}$, ambient temperature of $10^{4} \mathrm{~K}$, and ambient non-thermal pressure $\left(P_{B+C R}\right)$ equivalent to that of a $2.5 \mu \mathrm{G}$ effective magnetic field. Based on the $\mathrm{H}$ and $\mathrm{H}^{+}$vertical distributions, the choice of $n$ corresponds to $z=1000 \mathrm{pc}$. A variety of ionization conditions appear in the halo gas, but whether the warm phase or the hot phase is more plentiful is not well determined. The choices of ambient thermal and non-thermal pressures used here apply if the halo is cool and dominated by non-thermal pressures (see Boulares and Cox, 1990).

Although the abundances of gas-phase metals in the halo are not well determined, the halo gas-phase abundances may be closer to solar than are those in the disk (Savage and Sembach, 1996). Solar abundances are often used in SNR simulations of disk remnants and were used in these calculations.

\section{Evolution and Predictions}

\section{Results for a Single SNR}

A cool shell forms between 2.5 and $5 \times 10^{5}$ yrs. Afterward, the shock front expands faster than the hot bubble. Between the shock front and the periphery of the hot bubble lies a "thick" (large $\Delta r$ ), expanding, cool, weakly compressed shell. The hot bubble continues to expand for some time after shell formation, reaching its maximum radius of about $140 \mathrm{pc}$ at about $2 \times$ $10^{6}$ years. The ensuing diminution is very slow. For example, the hot cavity extends to $\sim 80 \mathrm{pc}$ at $10^{7}$ years.

At $10,000 \mathrm{yrs}$, the remnant is filled with hot $\left(>10^{7} \mathrm{~K}\right)$ gas, but its ions are far from equilibrium. The atoms in the interior are ionizing up through the $\mathrm{C}^{3+}, \mathrm{N}^{4+}$, and $\mathrm{O}^{5+}$ states. As a result, very large column densities of $\mathrm{UV}$ ions exist in the young remnant. Their Doppler shifted velocities and thermal broadening can be large. Around the time of shell formation, the shock front becomes too weak to ionize the swept up ambient gas to the $\mathrm{C}^{3+}, \mathrm{N}^{4+}$, and $\mathrm{O}^{5+}$ states. Henceforth, these ions only derive from previously hot, recombining gas. Oxygen can recombine to $\mathrm{O}^{+4}$ before nitrogen recombines to $\mathrm{N}^{+3}$ or carbon recombines to $\mathrm{C}^{+2}$ and the gas cools before all of the carbon has recombined to the $\mathrm{C}^{+2}$ level. As a result, the $\mathrm{C}^{3+}$ extends out to greater radii in the cooling gas than does the $\mathrm{N}^{4+}$ or $\mathrm{O}^{5+}$, and at very late times resides in cooled gas. For example, some $\mathrm{C}^{3+}$ remains at 18 million years, though 
the SNR had already cooled to the temperature of the ambient medium a million years earlier. The column densities are only weakly dependent on the age of the SNR, particularly after the first $5 \times 10^{4}$ yrs. The time integrals of the number of UV ions contained by the SNR are $8 \times 10^{69} \mathrm{O}^{5+}$ seconds, $7 \times 10^{68} \mathrm{~N}^{4+}$ seconds, and $2 \times 10^{69} \mathrm{C}^{3+}$ seconds. Dividing by the time integral of the area covered by UV ions gives the time and impact parameter averaged column densities: $5 \times 10^{13} \mathrm{O}^{5+} \mathrm{cm}^{-2}, 5 \times 10^{12} \mathrm{~N}^{4+} \mathrm{cm}^{-2}$, and $1 \times 10^{13}$ $\mathrm{C}^{3+} \mathrm{cm}^{-2}$. The soft X-ray flux peaks within the first $10^{5} \mathrm{yrs}$. At the peak, a

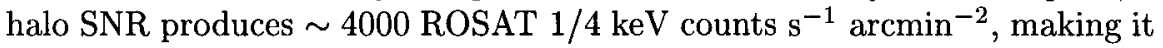
brighter than most areas within the northern and southern halos (Snowden et al., 1997). Early on, the ions are drastically underionized, with the ionization state varying within the remnant. But, the SNR cools faster than the X-ray ions can recombine. As a result, the gas becomes overionized and the spectra exhibit strong recombination edges (for example, the oxygen $739 \mathrm{eV}$ edge is prominent in the spectra by $2.5 \times 10^{5} \mathrm{yrs}$ ). Combining the time integral of the volume filled by hot $\left(T \geq 10^{6} \mathrm{~K}\right)$ SNR gas with the explosion rate from Ferrière (1995) gives a negligibly small filling fraction at $z=1000 \mathrm{pc}$.

\section{Estimates for the Population of Halo SNRs}

Most of the supernova explosions occur at lower heights than $z=1000$ pc and so are better confined by the greater ambient density and pressure. By running additional, computationally cheaper simulations for other $z$ 's, it was possible to estimate the fraction of volume filled by hot, halo SNR gas at several heights above the plane between about 300 and 2200 pc. The fraction is negligibly small. It is important to note that the volume filled by isolated SNR bubbles is much, much less than that outlined by SNR shock fronts because the radius to the shock front grows significantly beyond that to the bubble edge within the first $1 / 30$ of the remnant's life.

Because of the computational expense of using non-equilibrium ionization and recombination rates, the additional runs do not predict the time dependent UV ion and X-ray properties. Very approximate estimates of the time and space averaged column densities and emission fluxes of the ensemble of SNRs above $z \sim 300$ pc can be gotten by combining the supernova explosion rate with the predictions for a single SNR at $z \sim 1000 \mathrm{pc}$. The spatial distributions should be patchy. Using the UV ion-time integrals presented earlier, the predicted time and space average $\mathrm{O}^{5+}$ column density is $4.5 \times 10^{13} \mathrm{~cm}^{-2}$, which represents $3 / 4$ ths of the average observed column density (Hurwitz and Bowyer, 1996) after the Local Bubble contribution (Shelton and Cox, 1994) has been subtracted. The average $\mathrm{N}^{+4}$ and $\mathrm{C}^{+3}$ column densities from the simulations $\left(4 \times 10^{12} \mathrm{~cm}^{-2}\right.$ and $1 \times 10^{13} \mathrm{~cm}^{-2}$, respectively) provide smaller fractions of the observed totals. The gap may be due to photoionization and differences in ion production between this run and those at greater heights ${ }^{3}$. A halo SNR is rich in UV ions for its entire lifetime but is bright in soft

\footnotetext{
${ }^{3}$ The scale heights of $\mathrm{C}^{3+}$ and $\mathrm{N}^{4+}$ are greater than that of $\mathrm{O}^{5+}$.
} 
$\mathrm{X}$-rays for only the first $1 \times 10^{5} \mathrm{yrs}$. Thus, they cover much more of the sky with their UV ions than with their bright, soft X-ray emitting gas. The older halo SNRs contribute a patchy field of extremely dim X-rays having recombination edges and a softer spectrum. A rough estimate of the total X-ray contribution (found from the X-ray rates and the SN rate) is smaller than that found by Snowden et al. (1997), suggesting that either the model is in need of tweaking or other physical scenarios are also at work.

\section{Pressure Estimations and the Local Bubble}

The thermal pressure, $P_{t h}$, calculated from the observations of $\mathrm{O}^{5+}$ is much larger than that calculated from the $\mathrm{C}^{3+}$. There may be a natural, although scenario dependent, explanation. The simulations show that the $\mathrm{O}^{5+}$ lies in the hotter, thermal pressure dominated gas, while some of the $\mathrm{C}^{3+}$ lies in the cooler, non-thermal pressure dominated shell. As a result, $P_{t h}$ calculated from the $\mathrm{O}^{5+}$ emission and absorption predictions is much larger than that calculated from the $\mathrm{C}^{3+}$ results. This effect provides a method of estimating the lower limit to the non-thermal pressure $\left(P_{B+C R} \geq P_{t h}\left(\mathrm{O}^{5+}\right)-P_{t h}\left(\mathrm{C}^{3+}\right)\right)$.

Might the Local Bubble consist of the hot bubble of a SNR evolving in a low density, high non-thermal pressure medium? These simulations and those in Shelton (1996) show that until the cool shell forms, the SNRs are sufficiently X-ray bright and have $B$ to $C$ band ratios of $\sim 0.4$, in agreement with McCammon and Sanders (1990). Using $\mathrm{O}^{5+}$ as a criterion is more complicated because other sources (the Local Cloud and other clouds) may contribute. The $\mathrm{O}^{5+}$ column densities in the simulated SNRs are large enough to agree with Shelton and Cox (1994), but the $\mathrm{O}^{5+}$ velocities are too large to be observed by COPERNICUS (Jenkins, 1978) until after the shell forms.

Acknowledgements. Parts of this work were performed while the author held a National Research Council-NASA/GSFC Research Associateship.

\section{References}

Boulares, A., and Cox, D. P., (1990): ApJ 365, 544

Cioffi, D. F., (1991): In "The Interstellar Disk-Halo Connection in Galaxies", ed. H. Bloemen, p. 355

Ferrière, K. M., (1995): ApJ 441, 281

Hurwitz, M. and Bowyer, S., (1996): ApJ 465, 296

Jenkins, E., (1978): ApJ 219, 845

Maciejewski, W., Shelton, R. L., and Cox, D. P., (1996): AAS 188.4410M

McCammon, D., and Sanders, W. T., (1990): ARA\&A 28, 657

Savage, B. D., and Sembach, K. R., (1996): ARA\&A 34, 279

Shelton, R. L., (1996): Ph.D. Thesis, Dept. of Physics, Univ. of Wisconsin - Madison Shelton, R. L., and Cox, D. P., (1994): ApJ 434, 599

Snowden, S. L., Egger, R., Finkbeiner, D., Freyberg, M. J., and Plucinsky, P. P., (1997): submitted to ApJ 